\title{
A Robust Footprint Detection Using Color Images and Neural Networks
}

\author{
Marco Mora $^{1}$ and Daniel Sbarbaro ${ }^{2}$ \\ 1 Department of Computer Science, Catholic University of Maule, \\ Casilla 617, Talca, Chile \\ marco.mora@enseeiht.fr \\ 2 Department of Electrical Engineering, University of Concepcion, \\ Casilla 160-C, Concepcion, Chile \\ dsbarbar@die.udec.cl
}

\begin{abstract}
The automatic detection of different foot's diseases requires the analysis of a footprint, obtained from a digital image of the sole. This paper shows that optical monochromatic images are not suitable for footprint segmentation purposes, while color images provide enough information for carrying out an efficient segmentation. It is shown that a multiplayer perceptron trained with bayesian regularization backpropagation allows to adequately classify the pixels on the color image of the footprint and in this way, to segment the footprint without fingers. The footprint is improved by using a classical smoothing filter, and segmented by performing erosion and dilation operations. This result is very important for the development of a low cost system designed to diagnose pathologies related to the footprint form.
\end{abstract}

\section{Introduction}

When the foot is planted, not all the sole is in contact with the ground. The footprint is the surface of the foot in contact with the ground. The characteristic form and zones of the footprint are shown in figure 1(a). Zones 1, 2 and 3 correspond to regions in contact with the surface when the foot is planted; these are called anterior heel, posterior heel and isthmus respectively. Zone 4 does not form part of the surface in contact and is called footprint vault [18. These footprints play a key role in the detection of different foot's diseases.

The sole image can be acquired either in gray scale or color format. The segmentation of gray scale images can be done using standard techniques [7]. However, there are some problems with the segmentation of gray scale images produced by shadows, surface curvature and metamerism [21]. Taking in account the previous problems, segmentation techniques in color images have been developed. There are studies where the operators for edge detection have been extended from gray scales to color images [3], 8]. In other cases, segmentation techniques based on neural networks and statistical classifiers have been developed [1. Good reviews of segmentation techniques based on color images can be found in [4] and [5]. 
Among segmentation methods relevant for this study is the use of neural networks. In particular, the multilayer perceptron (MLP) and the training algorithm called backpropagation [9] have been successfully used in classification and functional approximation. An important characteristic of MLP is its capacity to classify patterns grouped in classes not linearly separable. Besides that, it has been shown that a one-hidden-layer perceptron (or two-layer perceptron) is an universal function estimator [19].

The first disadvantage of the backpropagation algorithm is its speed of convergence, this has led to the use of more sophisticated optimization methods. A good summary of these optimization methods is found in [13], and the application of such methods to the training of neural networks can be found in [17.

A second disadvantage of MLP trained with error backpropagation is that it may classify by mistake patterns not participating in the training process; i.e. it lacks of generalization. Generalization means that the neural network correctly classifies unknown patterns.

A technique to improve the generalization is called regularization, and consists in building a cost function from the sum of a function for error measurement (typically the average quadratic error) and a function representing the network complexity. Different regularization methods propose different functions for representing the network complexity, as example: weight decay 6], weight elimination [1] and approximate smoother [22. A current technique is the bayesian regularization, which uses the weight decay as the cost function, the Levenberg-Marquardt optimization algorithm [10, and a bayesian approach for defining the regularization parameters [2]. Among the advantages of the bayesian regularization technique are: (1) by using the Levenberg-Marquardt optimization algorithm, the speed of the learning process is improved, and (2) it provided the effective parameters the network is using. By using the network effective parameters, it is possible to define the amount of neurons in the hidden layer according to the procedure described in [10].

The data set used in this work, containing more than 200 images, was obtained using a prototype designed and built to capture sole images. Matlab, the Image Processing Toolbox and the Neural Networks Toolbox were used as platform for carrying out most of data processing work. The structure of this paper is as follows. Section 2 describes the problem of capturing footprints using gray scale images, shows the footprint segmentation using color images and neural networks, and describes the segmentation improvements. Section 3 shows a quality measurement of the footprint segmentation. Finally, Section 4 provides some conclusions.

\section{Footprint Segmentation Using Color Images and Neural Networks}

A first attempt to solve the segmentation problem considered gray scale images, since the use of this type of image allows the use of simple algorithms for its 


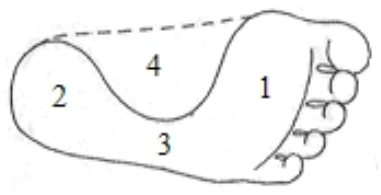

(a) Zones of the sole

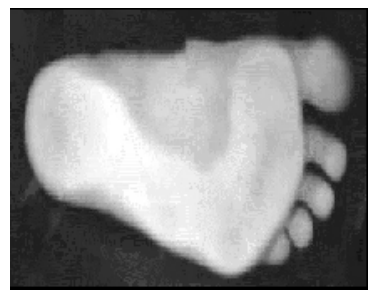

(b) Gray level image

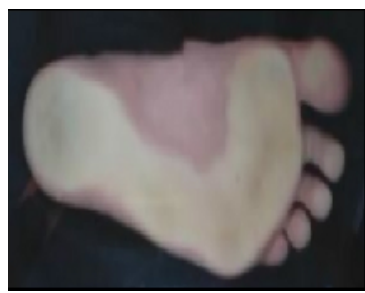

(c) Color image

Fig. 1. Images of the sole

segmentation. Figure 1(b) shows a gray-scale footprint image. It can be seen at first sight that there are patterns of the vault of the foot which have the same level of gray as others of the posterior heel. This means that different regions reflect the same amount of light (i.e. having the same gray values, whereas being differently colored), this phenomenon is known as metamerism [21. Thus, for this application, a segmentation based on gray scale is not adequate to separate the pixels of the footprint from the rest of the image by a simple threshold method.

Because of the metamerism problem in gray scale images, the use of color images is proposed. Color model means the specification of a system of threedimensional coordinates and a subspace of this system in which every color is represented by only one point 7 . The RGB color model has been used in this study. Figure 1(c) shows a color footprint image.

This work proposes the use of NN for footprint segmentation. The network acts as a pixel classifier [1, [15, and by the training process, it learns the pixel classes of the training set. In addition, by its generalization capabilities, it can also adequately classify pixels from the same image but not belonging to the training set and also pixels belonging to other images.

The neural network has three inputs corresponding to the RGB coordinates of the particular color. In the color footprint image in figure 1(c) it is clearly shown the existence of 3 pixel classes: the one from the image background, the one from the vault, and the one from the footprint. The network has an output assuming the value 1 for background pixels, 0 for the footprint, and -1 for the vault. The training set considers 709 samples selected from just one image, the $26 \%$ correspond to the background, $38 \%$ to the vault and $36 \%$ to the footprint. The size of each sample image is $434 \times 342$ pixels. The training of the multi-layer perceptron has the following characteristics:

- A hidden-layer MLP was used.

- Number of inputs: 3.

- Number of outputs: 1 .

- A bayesian regularization backpropagation as training algorithm.

- Learning in batch modality, where weights are updated at the end of each stage. 
Table 1. Determining the amount of neurons in the hidden layer for the footprint segmentation by using MLP

\begin{tabular}{|c|c|c|c|c|c|}
\hline NNCO & Epochs & SSE & SSW & Effective parameters & Total parameters \\
\hline 1 & $142 / 3000$ & $130.657 / 0.001$ & 55.7698 & $3.14 \mathrm{e}+000$ & 6 \\
2 & $45 / 3000$ & $0.00029 / 0.001$ & 8221.40 & $1.09 \mathrm{e}+001$ & 11 \\
3 & $18 / 3000$ & $0.00067 / 0.001$ & 6425.98 & $1.44 \mathrm{e}+001$ & 16 \\
4 & $36 / 3000$ & $0.00048 / 0.001$ & 7446.65 & $1.93 \mathrm{e}+001$ & 21 \\
5 & $125 / 3000$ & $0.00064 / 0.001$ & 3680.94 & $2.33 \mathrm{e}+001$ & 26 \\
6 & $66 / 3000$ & $0.00073 / 0.001$ & 3023.94 & $2.76 \mathrm{e}+001$ & 31 \\
7 & $113 / 3000$ & $0.00080 / 0.001$ & 3267.58 & $3.31 \mathrm{e}+001$ & 36 \\
8 & $329 / 3000$ & $0.00091 / 0.001$ & 3047.76 & $3.65 \mathrm{e}+001$ & 41 \\
9 & $104 / 3000$ & $0.00098 / 0.001$ & 2636.46 & $4.00 \mathrm{e}+001$ & 46 \\
10 & $150 / 3000$ & $0.00097 / 0.001$ & 2790.33 & $4.31 \mathrm{e}+001$ & 51 \\
11 & $140 / 3000$ & $0.00079 / 0.001$ & 2618.23 & $4.77 \mathrm{e}+001$ & 56 \\
12 & $292 / 3000$ & $0.00099 / 0.001$ & 2272.81 & $4.90 \mathrm{e}+001$ & 61 \\
13 & $194 / 3000$ & $0.00096 / 0.001$ & 2269.79 & $5.52 \mathrm{e}+001$ & 66 \\
14 & $218 / 3000$ & $0.00090 / 0.001$ & 2327.66 & $5.85 \mathrm{e}+001$ & 71 \\
15 & $182 / 3000$ & $0.00099 / 0.001$ & 2325.35 & $5.51 \mathrm{e}+001$ & 76 \\
16 & $225 / 3000$ & $0.00093 / 0.001$ & 2287.15 & $5.96 \mathrm{e}+001$ & 81 \\
30 & $261 / 3000$ & $0.00099 / 0.001$ & 2212.30 & $5.94 \mathrm{e}+001$ & 151 \\
\hline
\end{tabular}

- The initial network weights were generated by the Nguyen-Widrow method 12 because it increases the convergence speed of the training algorithm [10.

- The initial regularization parameters a and b were 0 and 1 respectively.

- Successive trainings were done increasing progressively the amount of neurons in the hidden layer.

To determine the amount of neurons of the hidden layer, the procedure described in [10] was used. The details of this procedure are shown in table 1, where NNCO corresponds to the number of neurons in the hidden layer, SEE is the sum of the quadratic errors and SSW is the sum of the weight squares.

From the previous table it can be seen that from 13 neurons in the hidden layer, the SSE, SSW and the effective parameters stay practically constants. As a result, 13 neurons are considered in that layer. The evolution of SSE, SSW and the effective parameters are shown in figure 2. Figure 3 shows the classification results. The classification errors in the footprint edge can be improved by carefully choosing with more detail the training set in this zone.

Because the detection of pathologies related to the footprint shape requires the capture of the footprint without toes, the previous result is improved by smoothing the footprint and by eliminating the toes.

The improvement steps are the following: (1) binarization, (2) footprint erosion in order to disconnect the toes if it is necessary, (3) smoothing of the footprint by median filter or a low pass filter in the frequency domain, (4) discharging the toes by ticketing and segmentation by size, and (5) image dilation in order to recover the size. The techniques previously noted are described in [7]. To visualize the improvements, the binarization is shown in figure $4(\mathrm{a})$, erosion is 


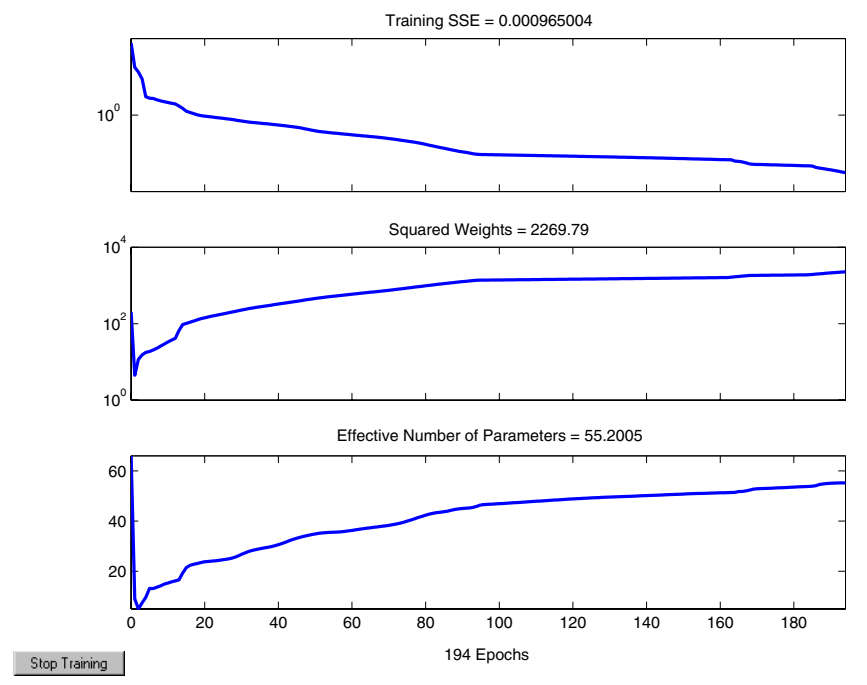

Fig. 2. MLP training

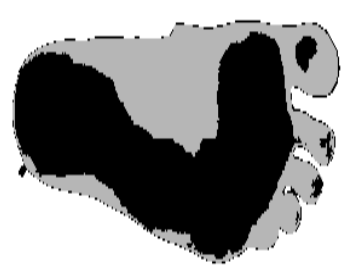

Fig. 3. Sole image classification

shown in figure 4(b), toe elimination is shown in figure 4(c), smoothing is shown in figure 4(d), dilation in figure 4(e), and the final result of the surrounding over the color image in figure $4(\mathrm{f})$.

\section{Quality Assessment of the Footprint Segmentation}

In the literature there are few methods to assess the quality of segmentation [14, 20], because the main reference is the one done by the human brain. Hence it is common in segmentation problems to compare the results obtained by the proposed algorithm with the human segmentation [15].

In order to assess the quality of the segmentation carried out by the MLP, a human-assisted segmentation was carried out for 10 footprint images and they are compared with the ones obtained by MLP. The results of such comparison are given in table 2, also the human segmentation of the footprint, the segmen- 


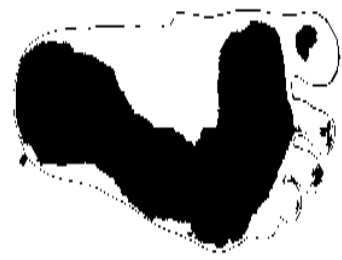

(a) Binarization

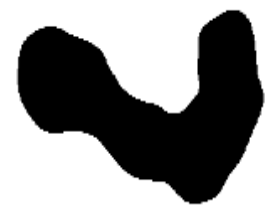

(d) Smoothing

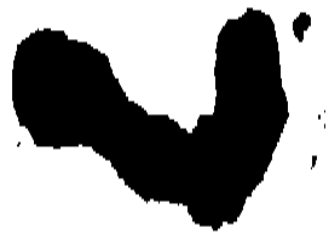

(b) Erosion

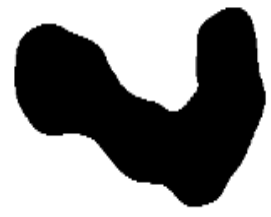

(e) Dilation

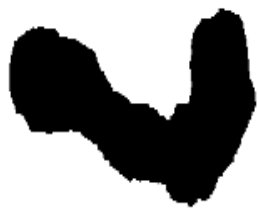

(c) Toe elimination

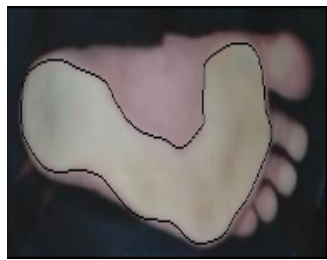

(f) Final edge

Fig. 4. Improvements in the footprint segmentation

Table 2. Quality assessment of the segmentation

\begin{tabular}{|c|c|c|c|c|}
\hline $\mathrm{N}$ & Size of images & Different colors & Pixels bad classified & Percent pixels good classified \\
\hline 1 & $324 \times 139$ & 12518 & 1827 & 95,94 \\
2 & $260 \times 112$ & 8741 & 1000 & 96,56 \\
3 & $260 \times 115$ & 8567 & 1335 & 95,46 \\
4 & $268 \times 121$ & 10008 & 1304 & 95,97 \\
5 & $280 \times 118$ & 10626 & 1029 & 96,87 \\
6 & $300 \times 138$ & 13062 & 1515 & 96,34 \\
7 & $280 \times 118$ & 7586 & 1005 & 96,96 \\
8 & $264 \times 113$ & 9903 & 709 & 97,62 \\
9 & $260 \times 118$ & 8244 & 1335 & 95,58 \\
10 & $294 \times 124$ & 10492 & 1337 & 96,25 \\
\hline
\end{tabular}

tation done by MLP and its errors are shown respectively in figures 5(a), 5(b) and 5(c). The figures show that the classification errors are concentrated in the borders. It must be noted that the footprint edges are not well defined and there is a small transition zone, where it is not possible to have a perfect human segmentation. It is possible to improve these results by using a training set with more samples corresponding to the edge zone. It is important to remark that the error introduced by the presence of toes is completely eliminated by the process described in the previous section. 


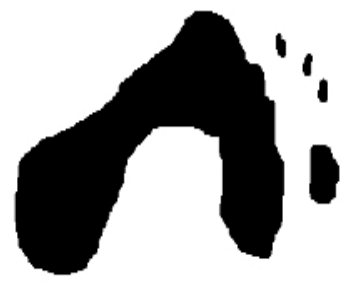

(a) Manual segmentation

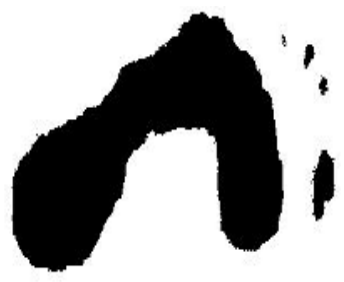

(b) MLP segmentation

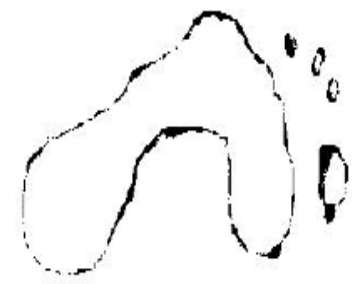

(c) Segmentation error

Fig. 5. Segmentation quality

\section{Conclusions}

This work has illustrated that the footprint segmentation using gray scales is not possible due to the problem known as metamerism, and the use of color image is then required.

The multilayer perceptron trained with bayesian regularization backpropagation not only enables to learn a training set representing the task of pixels classification but also to classify adequately pixels of other images.

Future work will consider a comparative study among different automatic segmentation algorithms, such as: non-parametric and non-supervised statistical classifier [23], self-organized neural networks [16], and the use of techniques for edge detection in color images [3, 8 .

The results of this study are promising and they have established a very simple and fast method for footprint automatic detection with no toes. It is foreseen on the near future the development of an automatic and real time diagnosis system of pathologies related with the footprint shape.

\section{References}

1. E. Littmann and H. Ritter, "Adaptive Color Segmentation: A Comparison of Neural Networks and Statistical Methods", IEEE Trans. on ANN, 81 , pp. 175-185, 1997.

2. D. Mackay. "Bayesian Interpolation", Computation and Neural System, California Institute of Technology, 1992.

3. T. Carron and P. Lambert, "Color Edge Detector Using Jointly Hue, Saturation and Intensity", in Proceedings IEEE International Conference on Image Processing, pp. 977-981, October 1994.

4. H. Cheng, X. Jiang, Y. Sun and J. Wang, "Color Image Segmentation: Advances and Prospects", Pattern Recognition, 34, pp. 2259-2281, 2001.

5. L. Lucchese and S. Mitra, "Color Image Segmentation: A State-of-the-Art Survey", (invited paper) Image Processing, Vision, and Pattern Recognition, Proc. of the Indian National Science Academy (INSA-A), New Delhi, India, 67A 2, pp. 207-221, 2001 .

6. G. Hinton, "Connectionist Learning Procedures", Artificial Intelligence, vol. 40, pp. 185-234, 1989. 
7. R. Gonzalez and R. Woods, "Digital Image Processing", Addison-Wesley, 1992.

8. R. Dony and S. Wesolkowski, "Edge Detection on Color Images Using RGB Vector Angle", in Proceedings of IEEE CCECE'99, Edmonton, Canada, 1999.

9. Rumelhart, McClelland, PDP group. "Explorations in Parallel Distributed Processing". The MIT Press. Vol. 1 y 2, 1986.

10. D. Foresee and M. Hagan, "Gauss-Newton Approximation to Bayesian Learning", Proceedings of the International Joint Conference on Neural Networks, 1997.

11. A. Weigend, D. Rumelhart and B. Huberman, "Generalization by WeigthElimination with Applications to Forecasting", Advances in Neural Information Processing System, vol. 3, pp. 875-872, 1991.

12. D. Nguyen and B. Widrow, "Improving the Learning Speed of 2-Layer Neural Networks by Choossing Initial Values of the Adaptive Weights", Proceedings of the IJCNN, vol. 3, pp. 21-26, 1990.

13. D. Luenberger, "Linear and Nonlinear Programming", second edition, AdissonWesley, 1984.

14. J. Liu, Y.-H. Yang, "Multiresolution Color Image Segmentation", IEEE Trans. On PAMI, 16(7),pp. 689-700, 1994.

15. J. Moreira and L. Da Fontuora, "Neural-Based Color Image Segmentation and Classification", Anais do IX SIBGRAPI, Brasil, 1996.

16. S. Haykin, "Neural Networks. A Comprehensive Foundation", second edition, Prentice Hall, 1999.

17. H. Demuht and M. Beale, "Neural Networks Toolbox for Use with Matlab: User Guide Version 4", 2003.

18. V. Valenti, "Orthotic Treatment of Walk Alterations", Panamerican Medicine, (in spanish)1979.

19. K. Funahashi. "On the Aproximate Realization of Continuous Mappings by Neural Network". Neural Networks, 2, 183-192, 1989.

20. M. Borsotti, P. Campadelli and P. Schettini, "Quantitative Evaluation of Color Image Segmentation Results", Patt. Rec. Lett., vol.19, 741-747, 1998.

21. T. Gevers and F. Groen, "Segmentation of Color Images", in Proceedings of 7th Scandinavian Conference on Image Analysis, 1991.

22. J. Moody and T. Rogntvalddson, "Smothing Regularizers for Proyective Basis Function Networks", Advances in Neural Information Processing System, vol. 9, pp. 585-591, 1997.

23. T. Hastie, R. Tibshirami and J. Friedman, "The Elements of Statistical Learning", Springer, 2001. 